\title{
CRescimento e Nutrição MIneral de Ipomoea hederifolia
}

\author{
Growth and Mineral Nutrition of Ipomoea hederifolia
}

GUZZO, C.D. ${ }^{2}$, CARVALHO, L.B. ${ }^{3}$, BIANCO, M.S. ${ }^{4}$ e BIANCO, S. ${ }^{5}$

\begin{abstract}
RESUMO - Um experimento em casa de vegetação foi conduzido entre novembro de 2005 e abril de 2006, na FCAV/UNESP, Brasil, objetivando estudar a produção de massa seca, a distribuição e o acúmulo de macronutrientes por Ipomoea hederifolia, uma importante planta daninha em culturas anuais e perenes no Brasil. As plantas foram cultivadas em vasos de $7 \mathrm{~L}$ com substrato de areia, que foram irrigados diariamente com solução nutritiva de Hoagland $\&$ Arnon. Os tratamentos corresponderam às épocas de avaliação, em intervalos de 14 dias, iniciando-se 21 dias após a emergência (DAE). Em cada avaliação, as plantas de quatro vasos foram analisadas quanto à produção de massa seca e ao conteúdo de macronutrientes. I. hederifolia apresentou pequeno acúmulo de massa seca e de macronutrientes no início da fase experimental, o qual foi intensificado após 63 DAE. As folhas foram as principais estruturas acumuladoras de massa seca na primeira metade do ciclo de $I$. hederifolia, enquanto os caules o foram na segunda metade. $\mathrm{N}$ e K foram os macronutrientes mais acumulados em plantas dessa espécie. O período de acúmulo máximo de massa seca e macronutrientes por I. hederifolia ocorreu entre 133 e 146 DAE.
\end{abstract}

Palavras-chave: plantas daninhas, corda-de-viola, massa seca, macronutrientes.

\begin{abstract}
A greenhouse experiment was carried out from November 2005 to April 2006 at FCAV/ UNESP, Brazil, to study the growth and accumulation and distribution of dry mass and macronutrients in Ipomoea hederifolia, an important weed in annual and perennial crops in Brazil. The plants were grown in 7-liter pots with sand substrate and irrigated with Hoagland and Arnon nutrient solution. The treatments corresponded to evaluation times, at 14 day intervals, beginning on the $21^{\text {st }}$ day after emergence (DAE). Plants of four pots were analyzed for growth and mineral nutrition. I. hederifolia showed small dry mass and macronutrient accumulation at the beginning of the experimental phase, intensified after 63 DAE. The leaves and stems were the main structures accumulating dry mass in the first and second half of the cycle, respectively,. N and K accumulated most in I. hederifolia plants. The maximum dry mass and macronutrient period for I. hederifolia occurred between 133 and 146 DAE.
\end{abstract}

Keywords: weeds, morningglory, dry mass, macronutrients.

\section{INTRODUÇÃO}

Ipomoea é o gênero que mais se destaca no âmbito da família Convolvulaceae, com 600-700 espécies no mundo. Entre as espécies do gênero, existem diversas plantas daninhas que são importantes em culturas anuais e perenes, destacando-se Ipomoea hederifolia, Ipomoea quamoclit, Ipomoea purpurea, Ipomoea triloba e Ipomoea nil, denominadas cordas-deviola (Kissmann \& Groth, 1999). Segundo esses autores, no Brasil, I. hederifolia apresenta vasta e expressiva ocorrência, sendo uma das espécies do gênero mais frequentemente

1 Recebido para publicação em 20.2.2010 e na forma revisada em 17.12.2010.

2 Mestrando em Agronomia (Produção Vegetal), Faculdade de Ciências Agrárias e Veterinárias, Universidade Estadual Paulista "Júlio Mesquita Filho" - FCAV/UNESP, Jaboticabal, Bolsista CNPq. ${ }^{3}$ Doutorando em Agronomia (Produção Vegetal), FCAV/ UNESP, Jaboticabal, Bolsista CNPq, <agrolbcarvalho@gmail.com>; ${ }^{4}$ Mestrando em Agronomia (Produção Vegetal), FCAV/UNESP, Jaboticabal. Bolsista CAPES; ${ }^{5}$ Professor Adjunto, Dep. de Biologia Aplicada à Agropecuária, FCAV/UNESP, Jaboticabal. 
encontradas nas áreas de produção agrícola. Atualmente, essa espécie tem sido crítica em plantações de cana-de-açúcar, milho, soja e outras culturas, tornando-se uma grande preocupação para os produtores brasileiros.

Considerando a importância atual de I. hederifolia como planta daninha, há necessidade de estudos básicos a respeito da biologia dessa espécie, envolvendo aspectos relacionados a reprodução, crescimento, desenvolvimento, exigências em nutrientes, respostas aos sistemas de controle e outros, visando obter informações que possam auxiliar no seu manejo. Segundo Bianco et al. (2004a,b), esses estudos básicos são fundamentais para entender o comportamento das plantas daninhas sob diferentes condições ambientais e para fornecer subsídios para predições de seu sucesso como infestante em função de novas práticas agrícolas e da introdução em novos ambientes.

Estudos a respeito de requerimentos nutricionais de plantas daninhas são de suma importância para a Ciência das Plantas Daninhas, pelo fato de que, segundo Pitelli (1985), a competição por nutrientes é um dos principais fatores ecológicos que alteram negativamente a produtividade das culturas agrícolas, assim como a competição por luz. Há trabalhos publicados a respeito de requerimentos nutricionais de plantas daninhas que destacam a importância desse tipo de estudo, podendo-se citar os de Rodrigues et al. (1995) com Commelina benghalensis, Erasmo et al. (2000) com Senna obtusifolia, Souza Filho et al. (2000) com Urena lobata, Gravena et al. (2002) com Hyptis suaveolens, Brighenti et al. (2003) com Cardiospermum halicacabum, Bianco et al. (2004a) com Rottboelia exaltata, Bianco et al. (2004b) com Desmodium tortuosum, Kazda et al. (2004) com Quercus petraea, Fagus sylvatica e Acer pseudoplatanus, Pedrinho Júnior et al. (2004) com Glycine maxe Richardia brasiliensis, Bianco et al. (2005a) com Indigofera hirsuta, Bianco et al. (2005b) com Brachiaria decumbens, Bianco et al. (2007) com G. max e Euphorbia heterophylla, Carvalho et al. (2007) com Zea mays e Brachiaria plantaginea, Bianco et al. (2008) com Senna obtusifolia, Duarte et al. (2008) com I. nil, Carvalho et al. (2009) com I. quamoclit e Bianco et al. (2010) com Solanum americanum.

O objetivo desta pesquisa foi estudar a produção de massa seca, a marcha de absorção e o acúmulo de macronutrientes por plantas de I. hederifolia cultivadas em condições padronizadas de nutrição mineral.

\section{MATERIAL E MÉTODOS}

O experimento foi conduzido em casa de vegetação do Departamento de Biologia Aplicada à Agropecuária, da Faculdade de Ciências Agrárias e Veterinárias - UNESP campus de Jaboticabal, SP, no período de novembro de 2005 a abril de 2006, utilizando vasos plásticos com capacidade para sete litros, tendo como substrato para crescimento areia de rio lavada e peneirada.

A semeadura foi feita em meados de novembro de 2005. Quando as mudas atingiram o estádio de dois pares de folhas verdadeiras totalmente expandidas, foi efetuado o desbaste, deixando-se apenas quatro plantas por vaso. Os vasos foram irrigados com solução nutritiva completa de Hoagland \& Arnon (1950), recebendo $25 \%$ da concentração original durante os primeiros 35 dias e, posteriormente, $50 \%$ até o final da fase experimental.

O delineamento experimental utilizado foi o inteiramente casualizado, com dez tratamentos e quatro repetições. Os tratamentos constituíram-se em épocas de avaliação, realizadas em intervalos de 14 dias, iniciandose aos 21 dias após a emergência (DAE), a saber: $21,35,49,63,77,91,105,119,133$ e 147 DAE. As repetições constituíram-se nos quatro vasos avaliados por época de avaliação.

Em cada época de avaliação, as plantas de quatro vasos foram coletadas e separadas em raízes, caules e folhas. A seguir, todo o material foi lavado conforme Sarruge \& Haag (1974), sendo submetido à rápida imersão em solução diluída de detergente neutro, lavagem por imersão em água destilada e, finalmente, imersão em água deionizada. Após lavagem, as diferentes partes das plantas foram acondicionadas em sacos de papel devidamente identificados e perfurados, para posterior secagem em estufa de renovação forçada de ar a $60-70{ }^{\circ} \mathrm{C}$ por 96 horas.

A massa seca foi obtida pesando-se o material seco em balança eletrônica com precisão de centésimos de gramas. Após a pesagem, o material foi moído em micromoinho tipo 
Willey, em malha de $20 \mathrm{~mm}$, e armazenado em frascos de vidros hermeticamente fechados. Após a moagem do material vegetal, foram determinados os teores de macronutrientes.

$\mathrm{O}$ nitrogênio total ( $\mathrm{N}_{\text {total }}$ ) e o fósforo $(\mathrm{P})$ foram determinados pelos métodos semi-micro kjedahl e colorimétrico do ácido fosfovanadatomolíbdico, respectivamente, conforme descrito por Sarruge \& Haag (1974). Na extração do potássio $(\mathrm{K})$, do cálcio $(\mathrm{Ca})$ e do magnésio $(\mathrm{Mg})$ foi utilizado o método descrito por Jorgensen (1977), por meio de espectrofotometria de absorção atômica. O S foi determinado pelo método turbidimétrico, descrito por Vitti (1989).

Os acúmulos de nutrientes para as diferentes partes da planta foram descritos multiplicando-se o teor do nutriente pela massa seca correspondente. $O$ acúmulo total foi obtido por meio do somatório dos acúmulos das diferentes partes da planta, enquanto o teor total da planta foi obtido pela relação entre o acúmulo total da planta e a massa seca total correspondente.

Para estimar o acúmulo total de massa seca e macronutrientes, utilizou-se a equação exponencial $y=\exp \left(a+b x+c x^{2}\right)$, sendo $y$ o acúmulo de massa seca e macronutriente e $x$ os dias após a emergência. As curvas foram ajustadas utilizando-se o software Statistica 6.0 (Statsoft, 2006), em função dos dias do ciclo de vida da planta, refletindo um esboço comportamental da espécie em relação ao nutriente estudado.

Os pontos de acúmulo máximo de massa seca e macronutrientes foram determinados pelas derivadas primeira e segunda da equação ajustada aos dados obtidos, utilizando o software Maple V (Char et al., 1991).

\section{RESULTADOS E DISCUSSÃO}

O acúmulo de massa seca por I. hederifolia foi lento no início do período experimental, atingindo o valor máximo teórico de 29,69 g por planta próximo aos 139 DAE (Figura 1). Após esse período, houve redução na massa seca acumulada pela planta daninha. Essa redução pode ter ocorrido devido à senescência de folhas, como afirmaram Duarte et al. (2008) e Carvalho et al. (2009) após observarem comportamento semelhante em plantas de $I$. nil e I. quamoclit, respectivamente.
Comparando os dados das três Ipomoea spp. já estudadas, pode inferir-se que, no período de maior acúmulo de massa seca por I. hederifolia (139 DAE), tanto I. nil quanto I. quamoclit apresentaram menores acúmulos de massa seca. Contudo, I. nil apresentou máximo acúmulo de $33,56 \mathrm{~g}$ por planta aos $123 \mathrm{DAE}$, e I. quamoclit, 6,46 g por planta aos 146 DAE. Isso evidencia que, entre as três espécies, a sequência crescente em porte máximo é I. nil, I. hederifolia e I. quamoclit.

Foi verificado grande acúmulo de massa seca de 77 a 133 DAE (Figura 1), assim como observado por Carvalho et al. (2009) em I. quamoclit. Duarte et al. (2008) também observaram maior acúmulo de massa seca iniciando-se após $77 \mathrm{DAE}$ em plantas de I. nil. Nesse periodo, esse grande aumento na massa seca de $I$. hederifolia ocorreu principalmente em função do acúmulo nos caules (Figura 2). Esse fato também foi observado em I. nil e I. quamoclit, podendo ser explicado em razão de essas espécies apresentarem hábito de crescimento trepador com longos caules (Lorenzi, 2000). Além disso, o ciclo de vida dessas espécies é mais prolongado que o de culturas anuais, acarretando problemas na colheita e perdas indiretas quando não controladas (Carvalho et al., 2009).

Dividindo o período experimental em três partes, a porcentagem de acúmulo de massa seca em folhas foi maior que nas outras

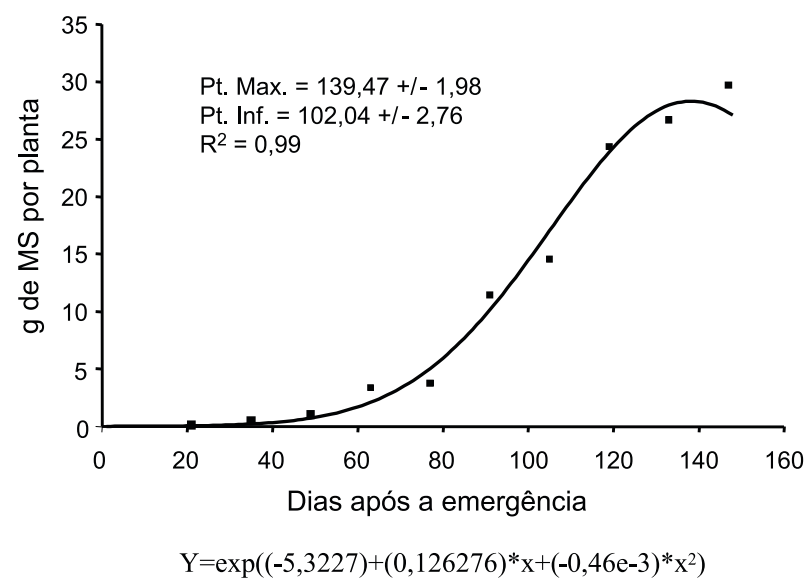

Figura 1 - Acúmulo de massa seca por Ipomoea hederifolia ao longo do período experimental, equação ajustada, respectivos pontos de máximo e inflexão e +/- desvio-padrão da média das quatro repetições. 
estruturas da planta na primeira parte, enquanto a porcentagem em caules foi maior na terceira parte do período experimental (Figura 2). Esse fato também foi verificado por Duarte et al. (2008) e Carvalho et al. (2009), sendo esse comportamento atribuído ao hábito de crescimento das espécies.

A porcentagem de acúmulo de massa seca nas raízes foi um pouco maior no início do período experimental, decrescendo à medida que a planta crescia (Figura 2). Esse fato também foi observado em I. nil, I. quamoclit e outras espécies já estudadas. Esse leve aumento pode ocorrer para permitir à planta fixar-se ao substrato, aumentando o contato das raízes com os nutrientes, e assim promover sua mais rápida absorção (Carvalho et al., 2007).

A porcentagem de acúmulo de massa seca nos caules aumentou substancialmente após $49 \mathrm{DAE}$, em detrimento principalmente das folhas de I. hederifolia (Figura 2). Isso permitiu maior porcentagem de acúmulo de massa seca nos caules do que nas outras estruturas da planta de 91 a 147 DAE. Segundo Carvalho et al. (2009), isso pode ser explicado pelo hábito de crescimento das espécies, as quais têm caules que crescem rapidamente, permitindo à planta buscar luz necessária à fotossintese.

A sequência de macronutrientes com mais altos teores em plantas de I. hederifolia foi $\mathrm{K}>\mathrm{N}>\mathrm{Ca}>\mathrm{Mg}>\mathrm{P}>\mathrm{S}$, com valores variando, para mais ou para menos, ao longo do período experimental (Tabela 1). Aos 77 DAE, foram verificados os maiores teores de $\mathrm{N}$ e K. Contudo, os maiores teores de $\mathrm{P}, \mathrm{Ca}, \mathrm{Mg}$ e $\mathrm{S}$ foram observados aos 49, 91, 63 e $133 \mathrm{DAE}$, respectivamente. Estudando nutrição mineral de plantas daninhas de folhas largas, Bianco et al. (2004b, 2005a, 2007, 2008) e Pedrinho Júnior et al. (2004) observaram que $\mathrm{N}$ e $\mathrm{K}$ foram os macronutrientes presentes em maiores quantidades em plantas de $D$. tortuosum, I. hirsuta, E. heterophylla, S. obtusifolia e R. brasiliensis, respectivamente. Duarte et al. (2008) e Carvalho et al. (2009) também relataram maiores teores de $\mathrm{N}$ e $\mathrm{K}$ em plantas de $I$. nil e I. quamoclit, respectivamente. Esse fato evidencia que $\mathrm{N}$ e $\mathrm{K}$ são os macronutrientes mais exigidos por essas plantas daninhas já estudadas.

$\mathrm{O}$ acúmulo de macronutrientes em plantas de I. hederifolia foi pequeno no início, aumentando após a primeira parte do período experimental para $\mathrm{N}, \mathrm{P}$ e $\mathrm{K}$, e pouco antes da metade do período experimental para $\mathrm{Ca}, \mathrm{Mg}$ e S (Figura 3). Um grande acúmulo de N, P e K foi verificado de 63 a $119 \mathrm{DAE}$; para Ca e S isso ocorreu de 77 a $119 \mathrm{DAE}$, e para $\mathrm{Mg}$, de 77 a 133 DAE. A planta de $I$. hederifolia acumulou o valor máximo estimado de 723,75 mg de $\mathrm{N}$ por planta aos $139 \mathrm{DAE}, 46,06 \mathrm{mg}$ de $\mathrm{P}$ por planta aos $146 \mathrm{DAE}, 810,02 \mathrm{mg}$ de $\mathrm{K}$ por planta aos $139 \mathrm{DAE}, 349,87 \mathrm{mg}$ de Ca por planta aos $140 \mathrm{DAE}, 148,03 \mathrm{mg}$ de Mg por planta aos $133 \mathrm{DAE}$ e $64,43 \mathrm{mg}$ de $\mathrm{S}$ por planta aos 140 DAE.

Tabela 1 - Teor de macronutrientes na planta de Ipomoea hederefolia ao longo do período experimental

\begin{tabular}{|c|c|c|c|c|c|c|}
\hline \multirow{2}{*}{ DAE } & $\mathrm{N}_{\text {total }}$ & $\mathrm{P}$ & $\mathrm{K}$ & $\mathrm{Ca}$ & $\mathrm{Mg}$ & $\mathrm{S}$ \\
\cline { 2 - 7 } & \multicolumn{6}{|c|}{$\left(\mathrm{g} \mathrm{kg}^{-1}\right.$ de massa seca $)$} \\
\hline 21 & 30,83 & 1,98 & 38,20 & 11,00 & 5,40 & 1,74 \\
\hline 35 & 23,20 & 1,56 & 28,55 & 9,26 & 4,27 & 1,44 \\
\hline 49 & 34,02 & 2,46 & 47,87 & 11,92 & 5,36 & 1,56 \\
\hline 63 & 33,77 & 1,62 & 45,76 & 13,19 & 6,54 & 1,87 \\
\hline 77 & 36,17 & 2,22 & 49,77 & 12,46 & 5,68 & 1,45 \\
\hline 91 & 32,52 & 2,14 & 39,43 & 12,76 & 5,70 & 2,21 \\
\hline 105 & 35,37 & 1,74 & 42,74 & 12,66 & 5,46 & 1,54 \\
\hline 119 & 30,12 & 2,05 & 30,99 & 11,21 & 5,49 & 2,48 \\
\hline 133 & 25,93 & 1,85 & 31,80 & 12,46 & 5,39 & 2,51 \\
\hline 147 & 27,46 & 1,87 & 26,66 & 9,76 & 4,18 & 2,17 \\
\hline
\end{tabular}

Figura 2 - Distribuição percentual da massa seca nas diferentes partes da planta de Ipomoea hederifolia ao longo do período experimental.

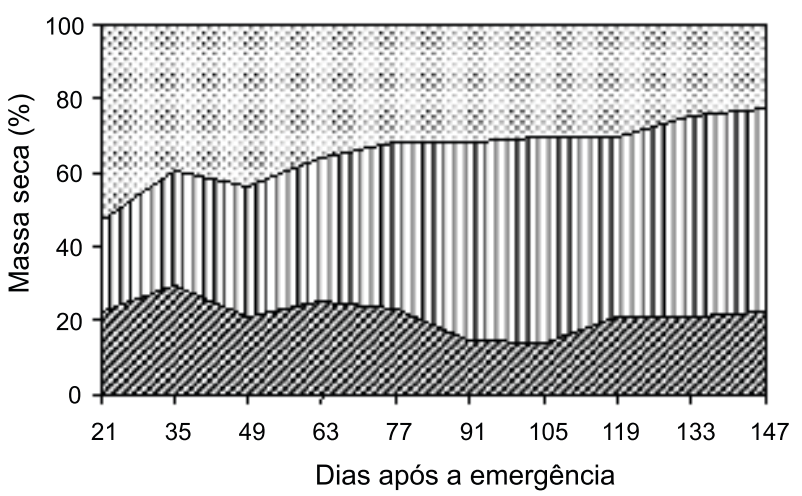

ØRAIZES $\square$ CAULES FOLHAS 

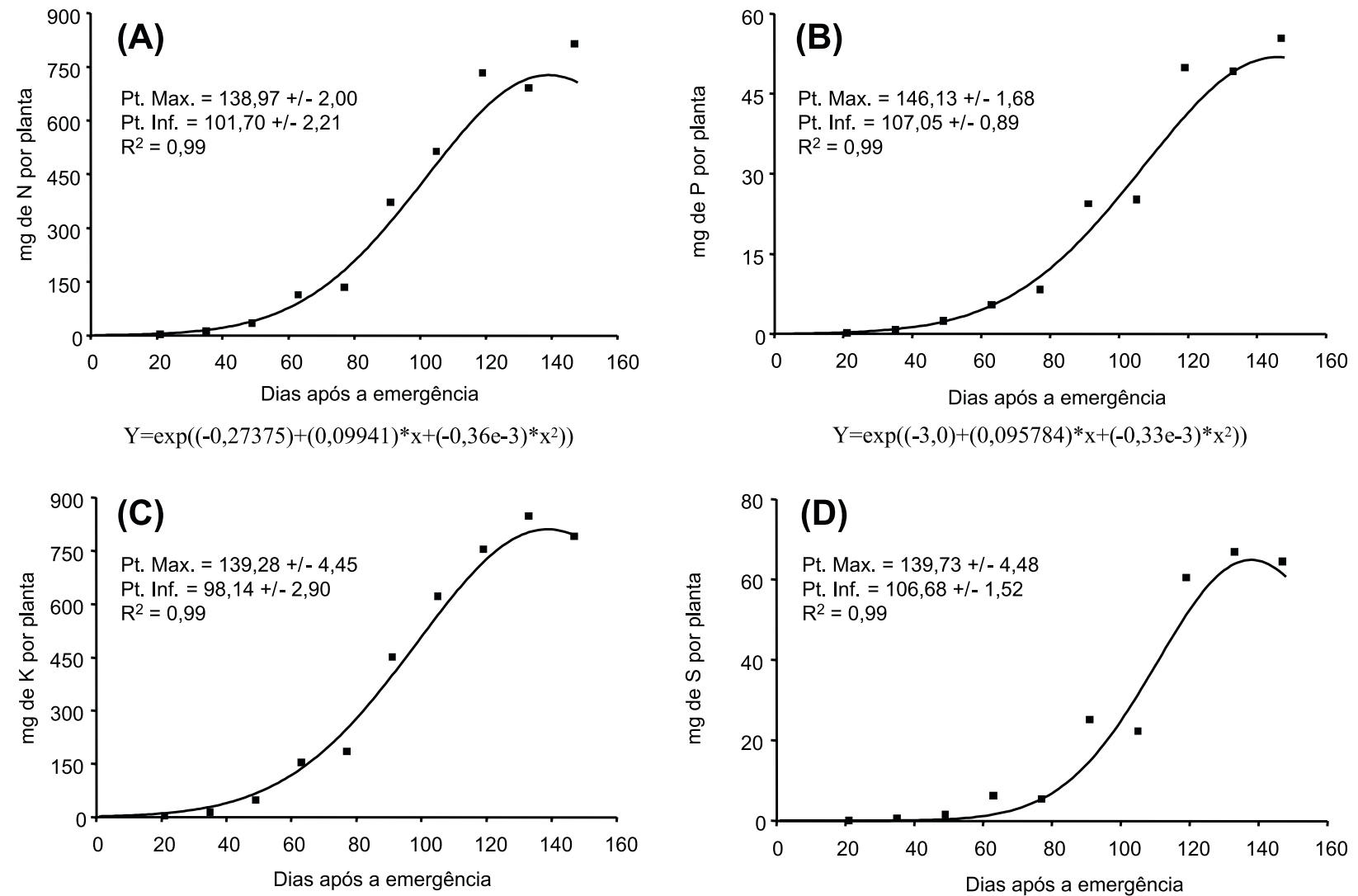

$\left.\mathrm{Y}=\exp \left((0,810826)+(0,085437) * \mathrm{x}+(-0,31 \mathrm{e}-3) * \mathrm{x}^{2}\right)\right)$
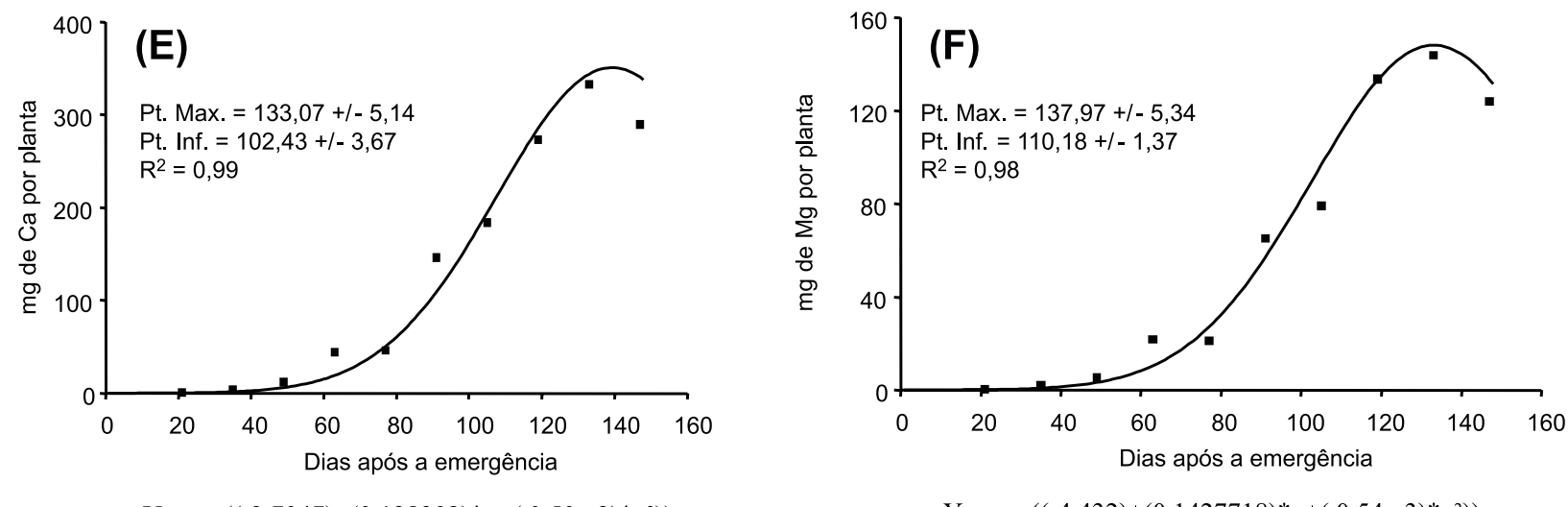

$\left.\mathrm{Y}=\exp \left((-3,7047)+(0,138302) * \mathrm{x}+(-0,50 \mathrm{e}-3)^{*} \mathrm{x}^{2}\right)\right)$

$\left.\mathrm{Y}=\exp \left((-4,432)+(0,1427718) * \mathrm{x}+(-0,54 \mathrm{e}-3) * \mathrm{x}^{2}\right)\right)$

Figura 3 - Curvas de acúmulos totais de nitrogênio (A), fósforo (B), potássio (C), cálcio (D), magnésio (E) e enxofre (F) por Ipomoea hederifolia ao longo do período experimental, equação ajustada, respectivos pontos de máximo e inflexão e +/- desvio-padrão da média das quatro repetições.

Comparando os dados das três Ipomoea spp. já estudadas, no ponto de acúmulo máximo, I. hederifolia acumulou mais macronutrientes que I. quamoclit $(167,62 ; 15,41 ; 149,46 ; 74,48$; 26,88; e 14,80 mg por planta de N, P, K, Ca, Mg e S, respectivamente). Além disso, I. hederifolia acumulou mais $\mathrm{K}, \mathrm{Mg}$ e $\mathrm{S}$ que I. nil $(780,63$; 139,68; e 38,71 $\mathrm{mg}$ por planta, respectivamente), porém menos $\mathrm{N}, \mathrm{Pe} \mathrm{Ca}(791,79 ; 50,82$; e $362,96 \mathrm{mg}$ por planta, respectivamente). Assim, pode-se evidenciar que I. hederifolia é mais exigente em macronutrientes que 
I. quamoclit e mais exigente em $\mathrm{K}, \mathrm{Mg}$ e $\mathrm{S}$ e menos exigente em N, P e Ca que I. nil.

Os macronutrientes mais acumulados em plantas de I. hederifolia foram aqueles que apresentaram maiores teores. A sequência de acúmulo foi $\mathrm{K}>\mathrm{N}>\mathrm{Ca}>\mathrm{Mg}>\mathrm{S}>\mathrm{P}$. Bianco et al. (2004a,b, 2007) e Pedrinho Júnior (2004), estudando plantas daninhas de folhas largas, e Duarte et al. (2008) e Carvalho et al. (2009), estudando Ipomoea spp., verificaram que $\mathrm{N}$ e $\mathrm{K}$ foram os macronutrientes mais acumulados nessas espécies. Eles são também os macronutrientes mais exigidos pela maioria das culturas agrícolas de interesse econômico (Epstein \& Bloom, 2005). Assim, a competição por macronutrientes exercida por plantas de I. hederifolia pode ser um importante fator biótico que altera negativamente o crescimento, o desenvolvimento e a produção das culturas.

Bianco et al. (2004a,b, 2007) relataram que o período de maior competição entre plantas daninhas e culturas anuais no Brasil fica em torno de $77 \mathrm{DAE}$. Isso em consequência da intensificação na assimilação de nutrientes pelas plantas (Carvalho et al., 2009), também observada nesta pesquisa. Durante esse período, uma planta de $I$. hederifolia acumulou 3,73 g de massa seca; 134,92 $\mathrm{mg}$ de $\mathrm{N} ; 8,29 \mathrm{~g}$ de P; 185,66 g de $\mathrm{K} ; 46,47 \mathrm{~g}$ de Ca; $21,21 \mathrm{~g}$ de $\mathrm{Mg}$; e 5,41 g de S. Comparando esses resultados com os obtidos para $I$. nil e I. quamoclit, pode-se afirmar que I. hederifolia é mais competitiva por macronutrientes que as demais Ipomoea spp. já estudadas aos 77 DAE, crescendo sob condições nutricionais semelhantes.

É importante evidenciar que, considerando a média dos valores dos pontos de inflexão e de acúmulo máximo estimado, a taxa de acúmulo diário de massa seca e de macronutrientes em $I$. hederifolia foi crescente até 104 DAE; o maior acúmulo de massa seca e de macronutrientes ocorreu aos 139 DAE. Esses dados permitem inferir que a competição mais intensa por macronutrientes pode ocorrer apenas em um estádio de desenvolvimento da cultura muito avançado, não sendo, portanto, um fator limitante ao desenvolvimento das culturas agrícolas anuais, principalmente.
Conclui-se que: as folhas são as principais estruturas acumuladoras de massa seca na primeira metade do ciclo de I. hederifolia, enquanto os caules o são na segunda metade; $\mathrm{N}$ e $\mathrm{K}$ são os macronutrientes mais acumulados em plantas de I. hederifolia; e o período de acúmulo máximo de massa seca e macronutrientes por $I$. hederifolia ocorre entre 133 e 146 DAE.

\section{LITERATURA CITADA}

BIANCO, S.; BARBOSA JÚNIOR, A. F.; PITELLI, R. A. Crescimento e nutrição mineral de capim-camalote. Planta Daninha, v. 22, n. 3, p. 375-380, 2004a.

BIANCO, S.; BARBOSA JÚNIOR, A. F.; PITELLI, R. A Crescimento e acúmulo de macronutrientes em Indigofera hirsuta. Ensaios. Ci., v. 9, p. 431-438, 2005a.

BIANCO, S.; PITELLI, R. A.; BELLINGIERI, P. A Crescimento e nutrição mineral de Desmodium tortuosum (Sw.) DC. Cult. Agron., v. 13, n. 1, p. 78-88, 2004 b.

BIANCO, S.; PITELLI, R. A.; CARVALHO, L. B. Estudo comparativo do acúmulo de massa seca e macronutrientes por plantas de Glycine max (L.) Merr. e Euphorbia heterophylla L. Ensaios Ci., v. 11, p. 61-72, 2007

BIANCO, S.; CARVALHO, L.B.; BIANCO, M.S. Growth and mineral nutrition of Solanum americanum. Planta Daninha, v.28, p.293-299, 2010.

BIANCO, S.; PITELLI, R. A.; CARVALHO, L. B. Crescimento e nutrição mineral de fedegoso. Ci. Cult., v. 3 , n. 1, p. $35-41,2008$

BIANCO, S.; TONHÃO, M. A. R.; PITELLI, R. A. Crescimento e nutrição mineral de capim-braquiária. Planta Daninha, v. 23, n. 3, p. 423-428, 2005 b.

BRIGHENTI, A. M.; VOLL, E.; GAZZIERO, D. L. P. Biologia e manejo do Cardiospermum halicacabum. Planta Daninha, v. 21, n. 2, p. 229-237, 2003.

CARVALHO, L. B. et al. Estudo comparativo do acúmulo de massa seca e macronutrientes por plantas de milho var. BR-106 e Brachiaria plantaginea. Planta Daninha, v. 25, n. 2, p. 293-301, 2007.

CARVALHO, L. B.; BIANCO, S.; PITELLI, R. A. Growth and mineral nutrition of Ipomoea quamoclit.

Planta Daninha, v. 27, n. 2, p. 283-288, 2009.

CHAR, B. W. et al. Maple V: language reference manual. New York: Springer-Verlag, 1991. 411 p.

DUARTE, D. J. et al. Crescimento e nutrição mineral de Ipomoea nil. Planta Daninha, v. 26, n. 3, p. 577-583, 2008 
EPSTEIN, E.; BLOOM, A. J. Mineral nutrition of plants principles and perspectives. 2.ed. Massachussets: Sinauer, 2005. $380 \mathrm{p}$.

ERASMO, E. A. L. et al. Efeito de níveis crescentes de calagem no crescimento e estado nutricional de fedegoso. Planta Daninha, v. 18, n. 2, p. 253-263, 2000.

GRAVENA, R. et al. Análise do crescimento de Hyptis suaveolens. Planta Daninha, v. 20, n. 2, p. 189-196, 2002.

HOAGLAND, D. R.; ARNON, D. J. The water culture method of growing plants without soil. Berkeley: University of California, 1950. 31 p. (Circular, 347).

JORGENSEN, S. S. Metodologia utilizada para análises químicas de rotina: guia analítico. Piracicaba: CENA, 1977. $24 \mathrm{p}$.

KAZDA, M. et al. Importance of mineral nutrition for photosynthesis and growth of Quercus petraea, Fagus sylvatica and Acer pseudoplatanus planted under Norway spruce canopy. Plant Soil, v. 264, n. 1, p. 25-34, 2004.

KISSMANN, K. G; GROTH, D. Plantas infestantes e nocivas. 2.ed. São Paulo: BASF, 1999. Tomo II. 978 p.

LORENZI, H. Plantas daninhas do Brasil: terrestres, aquáticas, parasitas e tóxicas. 3.ed. Nova Odessa: Instituto Plantarum, 2000. 608p.
PEDRINHO JÚNIOR, A. A. F.; BIANCO, S.; PITELLI, R. A. Acúmulo de massa seca e macronutrientes por plantas de Glycine max e Richardia brasiliensis. Planta Daninha, v. 22, n. 1, p. 53-61, 2004.

PITELLI, R. A. Interferências de plantas daninhas em culturas agrícolas. Inf. Agropec., v. 11, p. 16-27, 1985.

RODRIGUES, B. N.; PITELLI, R. A.; BELLINGIERI, P. A Efeitos da calagem do solo no crescimento inicial e absorção de macronutrientes por plantas de trapoeraba (Commelina benghalensis). Planta Daninha, v. 13, n. 1, p. 59-68, 1995.

SARRUGE, J. R.; HAAG, H. P. Análises químicas em plantas. Piracicaba: Escola Superior de Agricultura "Luiz de Queiroz", 1974. $56 \mathrm{p}$

SOUZA FILHO, A. P. S.; VELOSO, C. A. C.; GAMA, J. R N. Capacidade de absorção de nutrientes do capim-marandu (Brachiaria brizantha) e da planta daninha malva (Urena lobata) em função do pH. Planta Daninha, v. 18, n. 3, p. $443-450,2000$.

STATSOFT, INC. Data analysis software system. Version 6.0. Disponível em: $<$ http://www.statsoft.com $>$. Acesso em: 10 jan. 2010.

VITTI, G. C. Avaliação e interpretação do enxofre no solo e na planta. Jaboticabal: FUNEP, 1989. 37 p. 